\title{
A Parallel Study of Pigment Bleaching and Cytochrome Breakdown during Aging of Thylakoid Membranes
}

\author{
Jacques Dupont ${ }^{1}$ and Paul-André Siegenthaler ${ }^{2}$ \\ 1 Laboratoire de Biologie Végétale IV, CNRS (UA 1180), Université Pierre et Marie Curie, \\ 12 rue Cuvier, 75005 Paris, France \\ 2 Laboratoire de Physiologie Végétale, Université de Neuchâtel, chemin de Chantemerle 20, \\ CH-2000 Neuchâtel, Suisse
}

\begin{abstract}
Aging of freshly isolated thylakoid membranes from spinach leaves (Spinacia oleracea L.) leads to dramatic alterations in both the cytochrome ( $b_{559}$ (HP) and $f$ ) composition and pigment (chlorophylls $a$ and $b$ and $\beta$-carotene) content. These changes occur at a faster rate under anaerobic conditions or after heating thylakoid membranes, and in light as well as in darkness. In addition, when thylakoid membranes are heated at $78^{\circ} \mathrm{C}$ for $8 \mathrm{~min}$, or incubated in the presence of an emulsion of linoleic acid, a huge decrease in both cytochrome (particularly cyt. $b_{559}(\mathrm{HP})$ ) and pigment contents occur. Whatever the experimental conditions, cytochrome $b_{559}$ (HP) destruction occurs as soon the aging process starts. Conversely, pigment bleaching is detectable after an initial lag phase of about $60-70 \mathrm{~min}$. Then, the two processes (cytochrome breakdown and bleaching of pigments) appear to take place in parallel. The addition of salicylhydroxamic acid or 8-hydroxy-quinoline, two radical scavenger components, to the aging medium strongly reduces the rate and extent of cytochrome breakdown and pigment bleaching.

On the basis of these results, a tentative scheme accounting for the bleaching of pigments and the breakdown of cytochromes during aging in vitro of thylakoid membranes is proposed. It is suggested that these changes are mediated via a nonenzymatic mechanism in which free radicals could be implicated. The possible role of free radicals inducing ultrastructural changes at the level of chloroplast membranes in senescent leaves is also considered.
\end{abstract}

Key words: Aging - Cytochrome breakdown - Frce radical - Pigment bleaching - Spinacia oleracea - Thylakoid membrane.

Aging of isolated chloroplasts is known to cause severe impairements in both physiological functions and ultrastructural organization of thylakoid membranes. The most striking deterioration in the physiological functions are the irreversible decrease in cyclic and non-cyclic ATP synthesis, NADP+ photoreduction, apparent $\mathrm{O}_{2}$ evolution and proton pump activities (Siegenthaler 1969, Siegenthaler and Depéry 1977).

Beside these functional alterations, aging of isolated chloroplasts results also in structural changes of the photosynthetic membrane. There is a general swelling accompanied by a lack of light-induced shrinkage of thylakoids (Siegenthaler 1968, 1972) and a decrease in the number

Abbreviations: $\beta$-car., $\beta$-carotene; chl $a$, chlorophyll $a$; chl $b$, chlorophyll $b ; b_{559}$ (HP), high potential form of cytochrome $b_{550}$; 8-OH-Q, 8-hydroxy-quinoline; SHAM, salicylhydroxamic acid; RO; alkoxy radical of unsaturated fatty acids; ROO;, peroxy radical of unsaturated fatty acids, $\mathrm{ROOH}$, hydroperoxide of unsaturated fatty acids. 
of the chlorophyll protein bands concomitant with changes in the acyl composition (Henry et al. 1982). Aged chloroplasts undergo a breakdown of their endogenous lipids resulting in the release of large amounts of free unsaturated fatty acids (Constantopoulos and Kenyon 1968, Hoshina et al. 1975, Siegenthaler and Rawyler 1977). Furthermore, upon illumination, aged chloroplasts undergo peroxidative processes (Heath and Packer 1968, Hoshina et al. 1975).

Aging of thylakoid membranes is also characterized by a decrease in the intensity of chlorophyll $a$ fluorescence concomitant with an alteration in the photosystem II activity (Fragata 1975), as well as by a pigment bleaching. In this respect, it has been suggested that the chlorophyll bleaching occurring during the in vitro aging of chloroplasts from barley leaves is dependent on a protein called "chlorophyll oxidase", the activity of which could be due to the disorganization of thylakoid membranes taking place in the course of aging (Martinoia et al. 1982, Lüthy et al. 1984, Thomas et al. 1985).

Recently, it has been shown that thylakoid membranes aged in vitro for several hours display a dramatic decrease in the content of the high potential form of cytochrome $b_{559}$ (Dupont and Siegenthaler 1985). These authors have suggested that the destruction of this cytochrome could explain, at least in part, the loss of oxygen evolution and of electron flow through photosystem II observed during aging in vitro of spinach thylakoids (Siegenthaler 1969, 1972).

In the present study, the breakdown of cytochromes and the bleaching of pigments in thylakoid membranes were investigated during aging under well-controlled conditions in order to find out the relationship, if it exists, between these two degradative processes.

Results show that (1) these two processes occurred under both aerobic and strictly anaerobic conditions and (2) there was no experimental evidence for the involvement of a protein as previously suggested by Martinoia et al. (1982), Lüthy et al. (1984) and Thomas et al. (1985). In order to determine the involvement of free radicals in these processes, the effect of radical scavengers such as salicylhydroxamic acid and 8-hydroxyquinoline (Dupont 1983, Rustin et al. 1984) were examined. The results will be compared with the events taking place in senescent leaves.

\section{Materials and Methods}

Biological materials-Chloroplast thylakoid membranes were isolated from spinach leaves (Spinacia oleracea L.) obtained from a local market as previously described (Dupont and Siegenthaler 1985), suspended in $10 \mathrm{~mm}$ phosphate buffer ( $\mathrm{pH} 7.2$ ) and adjusted to about 70 to $80 \mu \mathrm{g}$ chlorophyll $(a+b) \cdot \mathrm{ml}^{-1}$.

Aging process-Thylakoid membranes were aged at room temperature $\left(20-25^{\circ} \mathrm{C}\right)$ in an

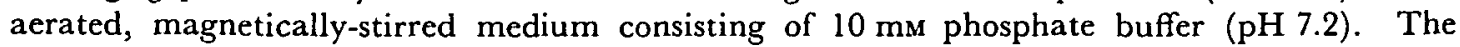
chlorophyll concentration varied but never exceeded $80 \mu \mathrm{g} \cdot \mathrm{ml}^{-1}$. At intervals, aliquots were used for cytochrome and pigment analysis.

Spectrophotometric studies-Changes in the pigment and cytochrome contents were recorded at room temperature with an Aminco DW 2A recording spectrophotometer using a $3 \mathrm{~nm}$ bandpath.

Qualitative and quantitative changes in pigments were followed by difference spectrophotometry between two thylakoid membrane suspensions at the same dilution, the aged one in the sample compartment, and the other one from the original thylakoid membrane suspension kept in ice and dark in the reference compartment. The resulting treated minus control difference spectra presented negative peaks located in the blue and red regions of the spectrum. Changes occurring in the red region of the spectrum $(646 \mathrm{~nm}$ and $678 \mathrm{~nm}$ ) originated from various forms of chlorophyll $b$ and $a$, respectively, as shown by Brody (1983). The spectral changes at $430 \mathrm{~nm}$, 
$456 \mathrm{~nm}$ and $489 \mathrm{~nm}$ were ascribed to various carotenes (Yamashita et al. 1969, Dupont et al. 1982a, b, Brody 1983).

Total cytochromes $\left(b_{559(\mathrm{HP})}+b_{563}+f\right)$ were determined at $561 \mathrm{~nm}$ in a dithionite-reduced minus ferricyanide oxidized spectrum. Cytochrome $f$ and the high potential form of cytochrome $b_{559}$ were determined at $554 \mathrm{~nm}$ and $559 \mathrm{~nm}$, respectively, from a hydroquinone-reduced minus ferricyanide oxidized difference spectrum. Calculations and difference extinction coefficients were given in the first paper of this series (Dupont and Siegenthaler 1985).

Total chlorophylls and $\beta$-carotene were determined without prior extraction of the pigments. The extinction coefficients, calculated in these conditions, were $52 \mathrm{~mm}^{-1} \cdot \mathrm{cm}^{-1}$ (at $678 \mathrm{~nm}$ ) and $56 \mathrm{~mm}^{-1} \cdot \mathrm{cm}^{-1}$ (at $646 \mathrm{~nm}$ ) for chlorophylls $a$ and $b$, respectively, and $85 \mathrm{~mm}^{-1} \cdot \mathrm{cm}^{-1}$ (at $489 \mathrm{~nm}$ ) for $\beta$-carotene. This rapid and accurate method for estimating the pigment contents of thylakoid membranes presented the advantage of avoiding several extraction operations, thus eliminating sampling errors, and measuring within the same biological sample not only the pigment but the cytochrome contents.

Lipoxygenase activity-Lipoxygenase activity was assayed from an acetone-extracted thylakoid preparation to rule out a possible inhibition of the activity by chlorophylls (Cohen et al. 1984). The rationale of such an assay has been reported elsewhere (Rustin et al. 1982). An assay with an acetone-treated purified soybean lipoxygenase (Type I from Sigma) was run in parallel as a control.

\section{Results}

Changes in cytochrome $\mathrm{b}_{559}(\mathrm{HP})$ and pigment contents under aerobic and anaerobic conditions-Table 1 shows that under aerobic conditions aging in vitro resulted in a decrease in the content of cytochrome $b_{559}$ (HP). In this experiment, only $47 \%$ of the initial content of this cytochrome was detected in 100 min-aged thylakoid membranes. During the same time, $11 \%$ and $4 \%$ of $\beta$ carotene and chlorophyll $a$ were bleached, respectively. Table 1 shows also that a more pronounced decrease in the content of cytochrome $b_{559}(\mathrm{HP})$ occurred under anaerobic than under aerobic conditions. Thus, more than $76 \%$ of the initial content of cytochrome $b_{559}$ (HP) was destroyed when aging proceeded in the absence of oxygen. In contrast, the extents of $\beta$ carotene and chlorophyll $a$ bleaching were similar to those found in the air-saturated aging medium. Whatever the experimental conditions (air-saturated or nitrogen-saturated media), there was no decrease in absorbance at $646 \mathrm{~nm}$, as could have been expected if chlorophyll $b$ would have been bleached (data not shown): Thus, under these mild experimental conditions,

Table 1 Effect of aerobic and anaerobic conditions on cytochrome and pigment contents during aging of thylakoid membranes

\begin{tabular}{|c|c|c|c|c|c|c|}
\hline \multirow{3}{*}{$\begin{array}{l}\text { Time } \\
\text { (min) }\end{array}$} & \multicolumn{6}{|c|}{ Contents $\left(\mathrm{nmol} \cdot \mathrm{ml}^{-1}\right)$} \\
\hline & \multicolumn{3}{|c|}{ Aerobic condition } & \multicolumn{3}{|c|}{ Anaerobic condition } \\
\hline & Cyt. $b_{559}(\mathrm{HP})$ & $\beta$-car. & Chl $a$ & Cyt. $b_{559}$ (HP) & $\beta$-car. & Chl $a$ \\
\hline 0 & $0.17 \pm 0.02$ & $14.9 \pm 2.3$ & $30 \pm 1.75$ & $0.17 \pm 0.02$ & $14.9 \pm 2.3$ & $30 \pm 1.75$ \\
\hline 100 & $0.08 \pm 0.01$ & $13.3 \pm 1.7$ & $28.9 \pm 1.5$ & $0.04 \pm 0.02$ & $13.6 \pm 1.7$ & $29 \pm 1.3$ \\
\hline
\end{tabular}

Quantitative estimation of cyt. $b_{559}$ (HP) and bleaching of pigments were carried out as described under Materials and Methods. Aerobic conditions refer to an aerated magnetically-stirred $\mathrm{O}_{2}$ saturated medium. Anaerobic conditions refer to a magnetically-stirred medium in a hermetically closed vessel, saturated with $N_{2}$. The values given represent the mean $\pm \mathrm{SD}$ of 3 independent experiments. 


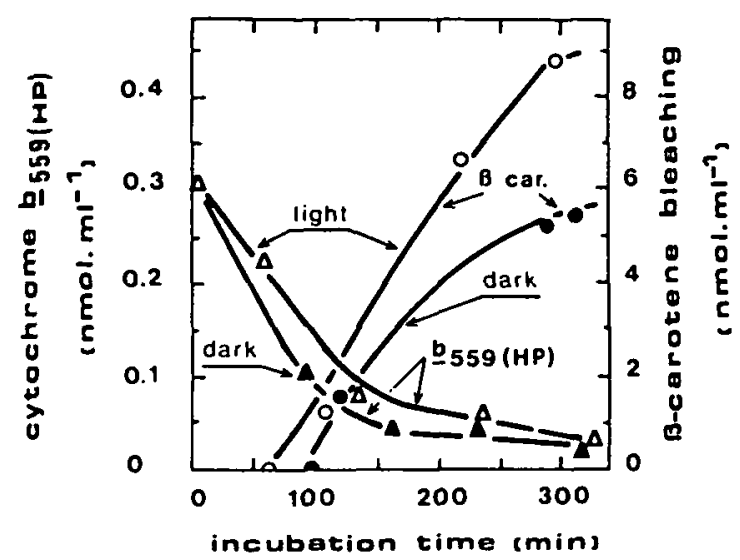

Fig. 1 Effect of dim light or darkness on cytochrome $b_{559}(\mathrm{HP})$ content and $\beta$-carotene bleaching in thylakoid membranes during aging. The aging process was carried out in an aerated medium. At 0 time, thylakoid membranes corresponded to $66 \mu \mathrm{g}$ chlorophylls $(a+b) \cdot \mathrm{ml}^{-1}, 15 \mu \mathrm{g} \beta$-carotene $\cdot \mathrm{ml}^{-1}$ and $0.31 \mathrm{nmol}$ cyt. $b_{559}(\mathrm{HP}) \cdot \mathrm{ml}^{-1}$. Dim light corresponded to $40 \mathrm{~W} \cdot \mathrm{cm}^{-2}$.

chlorophyll $b$ appeared more resistant toward aging than the other pigments, in agreement with other reports (Huber and Newman 1976, Brody 1983).

Influence of dim light or darkness on cytochrome and pigment contents-To obtain more informations on the mechanism(s) involved in the breakdown of cytochrome $b_{559}(\mathrm{HP})$ and pigment bleaching, the effect of aging was compared under light and dark conditions (Fig. 1). Results were similar under a wide range of light intensities (total energy from $40 \mathrm{~W} \cdot \mathrm{cm}^{-2}$ to $150 \mathrm{~W} \cdot \mathrm{cm}^{-2}$ ). It is noteworthy that cytochrome $b_{559}(\mathrm{HP})$ and $\beta$-carotene were destroyed under both dark and light conditions. Although light did not affect significantly the extent of cytochrome $b_{559(H P)}$ after an aging process of $300 \mathrm{~min}$, it increased the rate and extent of $\beta$-carotene bleaching. Moreover, upon illumination the initial lag phase was shortened. Chlorophyll $a$ was affected in a similar fashion under these aging conditions (data not shown). These results may suggest that cytochrome breakdown and pigment bleaching may be two related processes and that light would not be a prerequisite for the bleaching process.

Effect of dithionite on the dark system-In 1977 and 1978, Peiser and Yang reported that partially purified chlorophylls from spinach leaves were rapidly destroyed in vitro in the presence of bisulfite and linoleic acid hydroperoxide. In addition, there was no oxygen requirement. These authors suggested that the alkoxy radical which was formed from the reaction between $\mathrm{ROOH}$ and bisulfite was responsible for the destruction of chlorophylls through a free radical mechanism. To investigate the possible involvement of endogenous free radicals during aging in vitro of thylakoid membranes, the effects of an exogenous source of free radicals on the rates and extents of pigment and cytochrome breakdown was tested. For this purpose, thylakoid membranes were incubated in the dark and in the presence of dithionite.

Table 2 shows that after $120 \mathrm{~min}$ of dark-aging in the presence of dithionite, there were 6.5 times more $\beta$-carotene, 36 times more chlorophyll $a$ and 2 times more total cytochromes destroyed than in the control nitrogen-medium. Rather similar results were obtained when thylakoid membranes were incubated in dim light (data not shown). At the end of the incubation, and in the presence of dithionite, $50 \%, 53 \%$ and $54 \%$ of the initial content in $\beta$-carotene, chlorophyll $a$ and total cytochromes were respectively broken down, whereas only $7.5 \%, 1.5 \%$ and $21 \%$ of these compounds were destroyed in the control samples. Another feature is that under the dithionite conditions, $23 \%$ of the initial content in chlorophyll $b$ was bleached. After 17 hours of aging, more severe impairements in the pigment and cytochrome components were observed (results not shown): $83 \%$ of $\beta$-carotene, $81 \%$ of chlorophyll $a, 55 \%$ of chlorophyll $b$ and $78 \%$ of total cytochrome disappeared in the dithionite medium. Altogether, these data strongly point to a close relationship between the extent of bleaching of pigments and cytochrome 
Table 2 Effect of dithionite on pigment bleaching and on total cytochrome breakdown during aging of thylakoid membranes

\begin{tabular}{ccccc}
\hline Medium & $\beta$-carotene & $\begin{array}{c}\text { Chl } a \\
\left(\mathrm{nmol} \cdot \mathrm{ml}^{-1}\right)\end{array}$ & Chl $b$ & Total cytochromes \\
\hline Nitrogen & 1.4 & 0.7 & 0 & 0.11 \\
Dithionite & 9.3 & 25.2 & 7.5 & 0.22
\end{tabular}

Quantitative estimation of total cytochromes was carried out as described in Materials and Methods. Changes are expressed as chlorophylls and $\beta$-carotene bleached, and cytochromes destroyed after 120 min of aging in darkness. The nitrogen medium refers to a magnetically-stirred, $\mathbf{N}_{2}$-saturated medium in a hermetically closed vessel. Dithionite medium was made after addition of a few crystals at the beginning of the incubation. The vial containing the aging medium was introduced in a closed vessel with water saturated with dithionite. In this condition, the vessel was free-oxygen atmosphere. At 0 time, thylakoid membranes corresponded to $18.7 \mathrm{nmol} \cdot \mathrm{ml}^{-1} \beta$-carotene, $48 \mathrm{nmol} \cdot \mathrm{ml}^{-1}$ chlorophyll $a, 32.5 \mathrm{nmol} \cdot \mathrm{ml}^{-1}$ chlorophyll $b$ and $0.41 \mathrm{nmol} \cdot \mathrm{ml}^{-1}$ total cytochromes $(f+b$-type cytochromes).

breakdown. Moreover, and above all, these experiments suggest a possible involvement of free radicals in these two degradative processes. In order to test this hypothesis we have used exogenous radical scavengers with thylakoid preparations submitted to aging.

Effect of exogenous radical scavengers-Fig. 2 shows that an addition of SHAM or 8-OH-Q to the thylakoid membrane suspension stirred under aerated condition caused a drastic inhibition of both chlorophyll $a$ and $\beta$-carotene bleaching. In additicn, it is seen that the effect of these radical scavengers increased as a function of incubation time. The behavior of these inhibitors was similar on the breakdown of the total cytochrome (Table 3). In addition, these radical scavengers caused similar inhibitory effects under both aerobic and anaerobic conditions (data not shown).

All these data strongly suggest that the mechanism(s) which control cytochrome breakdown and pigment bleaching may share a common step which is affected by the same types of radical scavengers.

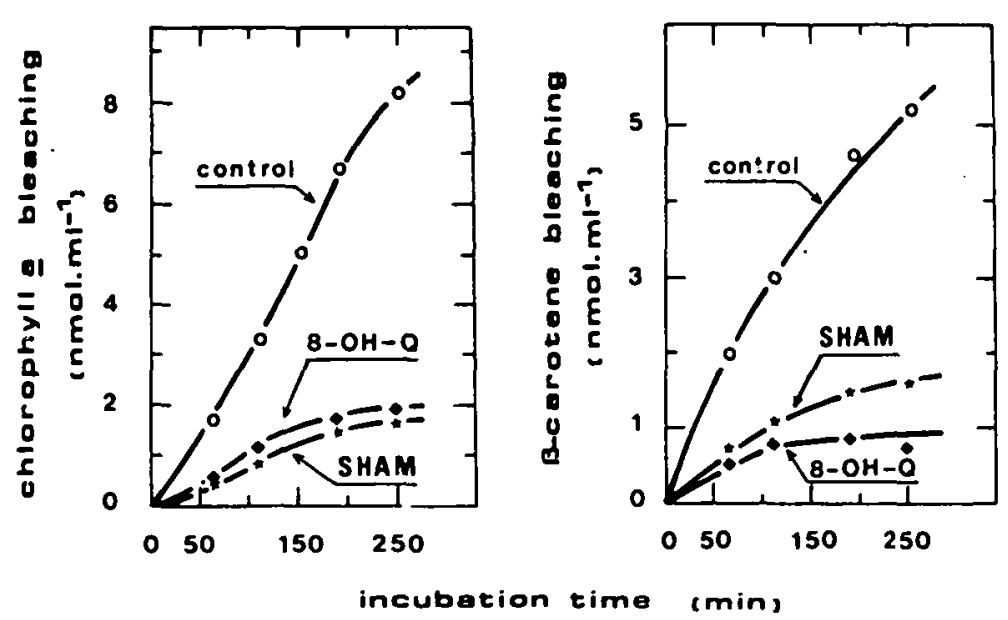

Fig. 2 Effect of radical scavengers on pigment bleaching during aging of thylakoid membranes. Aging was carried out as a function of time, in the presence or absence of SHAM and $8-O H-Q$ in $\operatorname{dim} \operatorname{light}\left(40 \mathrm{~W} \cdot \mathrm{cm}^{-2}\right)$ and under aerated conditions. At 0 time, thylakoid membranes contained $36 \mathrm{nmol} \cdot \mathrm{ml}^{-1}$ and $15 \mathrm{nmol} \cdot \mathrm{ml}^{-1} \mathrm{of} \mathrm{chlorophyll}$ $a$ and $\beta$-carotene, respectively. 
Effect of temperalure or added linoleic acid emulsion-When heat-treated thylakoid membranes were submitted to aging in vitro, the rates and extents of degradation of both cytochrome $b_{559}$ (HP) and cytochrome $f$ were much faster than those of the controls (Fig. 3A, B). For example, after $60 \mathrm{~min}$ of aging, roughly $98 \%$ of the initial content of cytochrome $b_{559}(\mathrm{HP}$ ) was destroyed in treated thylakoid membranes compared to only $28 \%$ in the control (Fig. $3 \mathrm{~A}$ ).
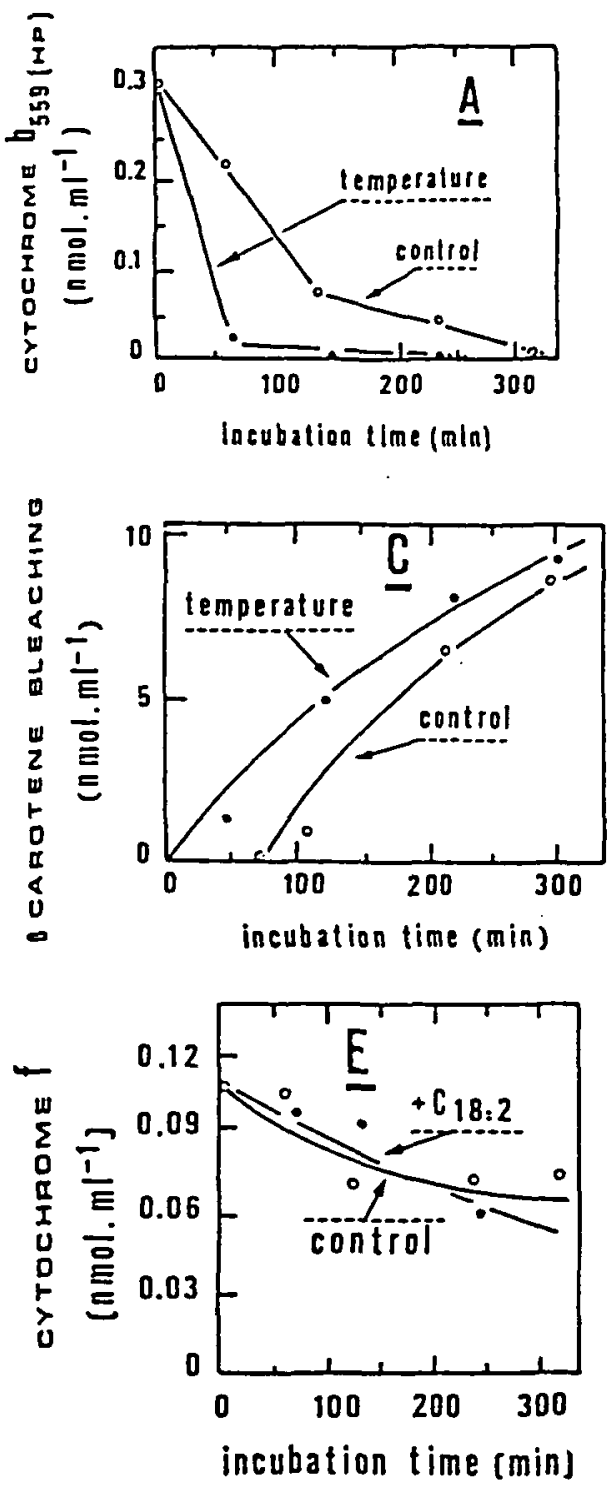
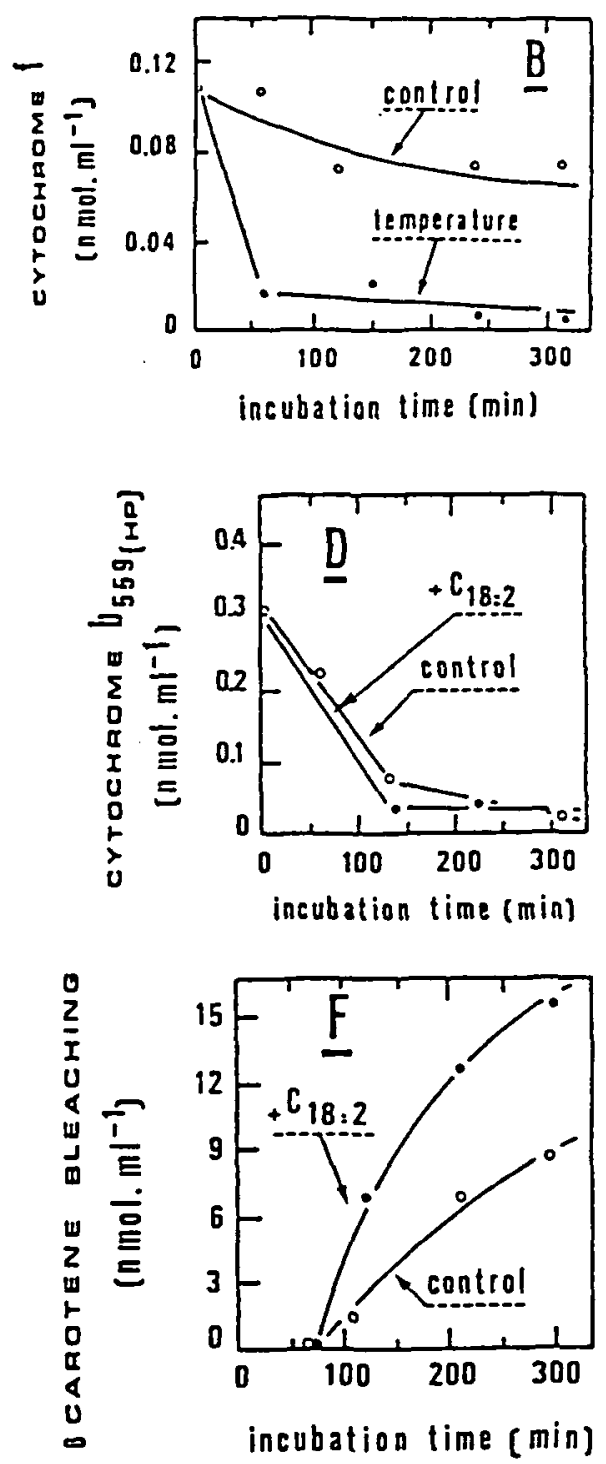

Fig. 3 Effect of temperature (A, B and C) or exogenous linoleic acid emulsion (D, E and F) on cyt. $b_{55 y}$ (HP) (A and $\mathrm{D})$, cyt. $f$ (B and $\mathrm{E}$ ) contents and $\beta$-carotene bleaching $(\mathrm{C}$ and $\mathrm{F})$ in thylakoid membranes during aging in vitro. $(\mathrm{A}, \mathrm{B}$ and $\mathrm{C})$ : Prior to the aging process, fresh thylakoid membranes were heated at $78^{\circ} \mathrm{C}$ for $8 \mathrm{~min}$. Aging was carried out in an aerated medium in dim light $\left(40 \mathrm{~W} \cdot \mathrm{cm}^{-2}\right)$. At 0 time, thylakoid membranes corresponded to $0.31 \mathrm{nmol}$ cyt. $b_{559}(\mathrm{HP}) \cdot \mathrm{ml}^{-1}, 0.12 \mathrm{nmol} \mathrm{cyt} \cdot f \cdot \mathrm{ml}^{-1}$ and $15 \mu \mathrm{g} \beta$-carotene $\cdot \mathrm{ml}^{-1}$. (D, E and F): Linoleic acid emulsion ( $0.21 \mu \mathrm{M})$ was added in the aging medium as an emulsion (Dupont et al. 1982a). At 0 time, cytochrome and pigment contents were the same as in A, B and C. 
Table 3 Effect of radical scavengers on cytochrome breakdown during aging of thylakoid membranes

\begin{tabular}{lcc}
\hline \multirow{2}{*}{ Inhibitor } & \multicolumn{2}{c}{ Total cytochrome broken down } \\
\cline { 2 - 3 } None & $\left(\mathrm{nmol} \cdot \mathrm{ml}^{-1}\right)$ & $(\%)$ \\
+SHAM (5 mM) & 0.16 & 53 \\
+8-OH-Q (5 mM) & 0.11 & 36 \\
& 0.10 & 33 \\
\hline
\end{tabular}

\begin{abstract}
Quantitative estimation of total cytochromes was carried out as described in Materials and Methods. Aging was performed in the absence or presence of radical scavengers in dim light, corresponding to $40 \mathrm{~W} \cdot \mathrm{cm}^{-2}$, during $110 \mathrm{~min}$ and under aerated conditions. Total remaining cytochromes were determined, at the end of the aging process, from room temperature difference spectra (dithionite anaerobic minus aerobic) as described by Dupont and Siegenthaler (1985). The amount of broken down cytochrome was calculated by comparison with that initially present in the thylakoid membranes $\left(0.30 \mathrm{nmol} \cdot \mathrm{ml}^{-1}(f+b\right.$-type $)$ cytochromes $)$. Percent values correspond to percent broken down compared to the control membranes at 0 time.
\end{abstract}

Similar behavior was observed with cyt. $f$. After $68 \mathrm{~min}$ of aging, about $81 \%$ of the initial content of this cytochrome was destroyed in heat-treated thylakoid membranes compared to only $7.5 \%$ in the control (Fig. 3B). Interestingly, similar observations were made for the bleaching of $\beta$-carotene (Fig. 3C) and chlorophyll $a$ (data not shown). In temperature-treated thylakoid membranes, the $\beta$-carotene content decreased immediately after the onset of aging whereas in the control sample the bleaching started only after an initial lag phase of about one hour (Fig. 3C). From this, one can conclude that the bleaching of $\beta$-carotene (and chlorophyll a) and the breakdown of cytochromes $b_{559}$ (HP) and $f$ are not under the control of a heat-labile factor. Rather, heat treatment enhanced considerably, and with a faster rate than in the controls, the breakdown of cytochromes and the bleaching of pigments.

The effects of an emulsion of linoleic acid on the extent of both the breakdown of cytochromes $b_{559}$ (HP) and $f$ and the bleaching of $\beta$-carotene are shown in figures $3 \mathrm{D}, \mathrm{E}$ and $\mathrm{F}$. An addition of a low amount of the emulsion of the unsaturated fatty acid $(0.2 \mu \mathrm{M})$ increased the rate but not the extent of cytochrome $b_{559(\mathrm{H})}$ breakdown. For example, in thylakoid membranes aged for 5 hours, the extents of the breakdown of cytochrome $b_{559}$ (HP) were similar in both treated and control membranes. Roughly, $90 \%$ of the initial content had disappeared during this period of time (Fig. 3D). In contrast, the rate and extent of cyt. $f$ breakdown were not enhanced in the presence of an identical concentration of the same emulsion of linoleic acid (Fig. 3E). This contrasts with the effects of a heat treatment of the thylakoid membranes on cyt. $f$ (see Fig. 3B). After an initial lag period of $60 \mathrm{~min}$ there was, in the linoleic acid-treated thylakoid membranes, a decrease of the $\beta$-carotene content which was complete after 5 hours of incubation compared to $69 \%$ in the control (Fig. 3F).

\title{
Discussion
}

The purpose of this discussion is to elucidate the mechanisms by which cytochromes and pigments are destroyed during aging in vitro. Our results clearly show that (1) bleaching of pigments and cytochrome breakdown are obviously two related phenomena, probably sharing some common step(s); (2) these changes occur in the light as well as in darkness, and under 
aerobic or anaerobic conditions; (3) it is likely that these changes are not under the control of a thylakoid-bound enzyme; (4) scavengers of free radicals strongly slow down the decrease in both cytochrome and pigment contents.

In this investigation we show that the pigment bleaching which occurs during aging in vitro of thylakoid membranes is mainly due to $\beta$-carotene and chlorophyll $a$. The content of chlorophyll $b$ remains rather constant during this process, except when thylakoid membranes are submitted to dithionite treatment (cf. Table 2). A preferential decrease in $\beta$-carotene and chlorophyll $a$ contents appear to be a general characteristic of senescence in green tissues (Huber and Newman 1976, Jenkins et al. 1981, Woolhouse 1984). Moreover, it has been reported to occur as a consequence of herbicide treatment in Scenedesmus cells (Van Rensen 1975), of $\gamma$ irradiation with Pisum sativum seeds (Merzlyak et al. 1984), of ozone fumigation in the light with spinach leaves (Sakaki et al. 1983) and of illumination in isolated chloroplasts upon illumination (Heath and Packer 1968).

The causes of pigment bleaching during aging in vitro of thylakoid membranes are controversial. In barley chloroplasts, Martinoia et al. (1982) and Lüthy et al. (1984) have suggested that two distinct enzymes (a peroxidase and a "chlorophyll oxidase) are directly involved in the bleaching of pigments. These enzymes which are tightly bound to the thylakoid membranes could be activated as a consequence of alterations in the structure of the photochemical apparatus. The bleaching activity geared by this "chlorophyll oxidase" is strictly oxygen-dependent and requires the presence of added linoleic acid (as an emulsion) or other unsaturated fatty acids (Lüthy et al. 1984). A boiled preparation of thylakoid membranes does not exhibit any bleaching activity (Martinoia et al. 1982).

The present study suggest an opposite (but not an exclusive) view. It is likely that the changes in pigments and cytochromes occurring during aging in vitro of thylakoid membranes are the result of a non-enzymatic process. This concept is based upon the observation that the rate and extents of aging-induced alterations of both pigments and cytochromes increase (but are not prevented) by a pretreatment of thylakoid membranes at $78^{\circ} \mathrm{C}$ for $8 \mathrm{~min}$ (see Fig. $3 \mathrm{~A}$, $B$ and $\mathrm{C}$ ). Quite obviously, if a heat labile factor had been involved in the deteriorative process, no (or only small changes) would have been detected in heat-treated membranes (Dupont et al. 1982a). Thus, it is unlikely that a putative "chlorophyll oxidase" is involved in the degradative changes related to aging of thylakoid membranes from spinach leaves. This is further strengthened by the observation that the deteriorative changes were not strictly dependent on oxygen (see Table I) as expected if a "chlorophyll oxidase" was involved.

However, this interpretation a priori does not rule out the possibility that lipoxygenase is involved in the bleaching process. Lipoxygenase activity has been detected in plastid fractions (Douillard 1971) and is known to be involved in the bleaching of chlorophyll $a$ and $\beta$-carotene (Imamura and Shimizu 1974, Weber and Grosch 1976, Ikediobi and Snyder 1977, Grossman et al. 1984). But unfortunately we failed to find any detectable lipoxygenase activity in thylakoid membranes from spinach leaves. The difference between our results and those reported by Douillard (1971) is due to the method used to measure lipoxygenase activity. Our assay is based on the enzymic determination of linoleic acid disappearance (substrate of the enzyme lipoxygenase) through the measurement of stoichiometric $\mathrm{O}_{2}$ uptake in an $\mathrm{O}_{2}$ electrode cell (Rustin et al. 1982), whereas Douillard (1971) estimated the activity by following the consumption of $\mathrm{O}_{2}$. This polarographic method has been shown to be inaccurate and to reflect, at least in part, an unspecific oxidation of hydroperoxides in the presence of membrane cytochromes (Dupont et al. 1982a). Thus the hypothesis that this enzyme is involved in chlorophyll $a$ and $\beta$-carotene bleaching during aging in vitro of thylakoid membranes can be rejected. This fits well with the fact that pigment bleaching as well as cytochrome breakdown take place in heattreated thylakoid membranes (see Fig. 3A, B and C). 
Another argument in favor of a non-enzymatic mechanism in pigment bleaching and cytochrome breakdown is brought about by the effects of SHAM and 8-OH-Q. These compounds are strong inhibitors of these two processes (see Fig. 2 and Table 2). These inhibitors presumably act as radicals complexing radicalar forms of the biological targets (Russell 1959, Ingold 1961, Walling 1963, Rabek and Ranby 1978, Dupont 1983), namely radicals of unsaturated fatty acids $(\mathrm{R} \cdot)$ or alkoxy radicals of unsaturated fatty acids ( $\mathrm{RO}^{\cdot}$ ), thus preventing their further reactivity (Klein et al. 1984). This proposal is further strengthened by the effect of added linoleic acid emulsion on the rates of cytochrome $b_{559(\mathrm{HP})}$ and $\beta$-carotene breakdown (see Fig. 3D and F). The non-deleterious effect of exogenous linoleic acid emulsion on cyt. $f$ (see Fig. $3 \mathrm{E}$ ), compared to the control, could be due to a non-accessibility of the unsaturated fatty acid (or of its radicalar form) to the cytochrome haem group as a consequence of an internal location of this cytochrome in normally oriented thylakoid membranes (Cox and Andersson 1981, Anderson et al. 1985). As previously shown (Dupont et al. 1982a), such linoleic acid emulsions inevitably contain low amounts of the unsaturated fatty acid hydroperoxide ( $\mathrm{ROOH})$ and presumably traces of radicals $\mathrm{R} \cdot$ which are precursors for the oxidized forms ( $\mathrm{RO} \cdot$ and $\mathrm{ROO} \cdot \mathrm{O}^{\circ}$ of unsaturated fatty acids (RH) (Egmond et al. 1973, Galliard and Chan 1980). This could explain why an addition of linoleic acid containing low amounts of radicals increases the rates and extents of $\beta$-carotene and chlorophyll $a$ bleaching and cyt. $b_{559}$ (H) breakdown (Fig. 3D, F). In addition, the dramatic disorganization in the structure and function of the thylakoid membrane caused by the penetration of low amounts of exogenous unsaturated fatty acids into the hydrophobic core of the thylakoid membrane is well established (Siegenthaler 1972, Okamoto and Kato 1977a, Okamoto et al. 1977b).

Another convincing argument in favor a probable involvement of a free radical mechanism in the cytochrome destruction and pigment bleaching processes is supported by the experiments carried out in the presence of dithionite. Together with its well known reducing properties, dithionite also acts as a producer of free radical compounds (G. Dodin, personnal communication). In this context, dithionite mimicks bisulfite by producing radicals which would react with chlorophylls ( $a$ and $b$ ) (and $\beta$-carotene) in a one-electron oxidation step leading to an accumulation of irreversible oxidation products (Peiser and Yang 1978). On the other hand, it is well known that, in the presence of peroxides, cytochromes may be degraded by opening of the tetrapyrolle ring, loss of the $a$-methene carbone with consequent loss of iron and catalytic activity (Falk 1964, Brown and Jones 1968).

Recently, Dupont and Siegenthaler (1985) reported that cyt. $b_{559}$ (HP) and cyt. $f$ present a differential sensitivity towards the aging process. Cyt. $f$ appeared more resistant than the other hemoprotein except after a heat-treatment of the thylakoid membrane suspension ( $c f$. Fig. 3B). This observation suggests that, due to a deep disorganization of the lipoprotein complexes of the thylakoid membranes, the haem group of cyt. $f$ which is well "buried" in the lipid matrix (Mansfield and Bendall 1984, Ortiz and Malkin 1985) may be more susceptible to free radical attack than in thylakoid membranes which have not been submitted to a heattreatment.

In the light of our results we propose that the formation, and the subsequent accumulation, of free radicals is the main factor involved in the destruction of cytochromes and pigments in aged-thylakoid membranes. This proposal is in agreement with reports by Peiser and Yang (1977, 1978), Takahama (1983) and Kato and Shimizu (1985). In that respect, it is noteworthy that allomerization of chlorophylls $a$ and $b$ proceeds via a free radical mechanism (Hynninen 1981, Merzliak and Koorighnih 1984). Although no attempt was made to characterize the products of $\beta$-carotene and chlorophylls breakdown, the fact that the specific absorption of the pigments decreased greatly throughout the visible range without the formation of new peaks, suggests that these pigments were rapidly converted into colorless products. 
Finally, the observation that bleaching of pigments and destruction of cytochromes occur independently under aerobic and anaerobic conditions, and in both light and darkness, may suggest a random attack by free radicals. The reaction sequence of this process as well as the identity of these radicals remain to be elucidated and are currently under investigation. Indeed it could be a clue to explain the ultrastructural changes of chloroplasts of senescent (Mittelheuser and Van Steveninck 1971, Huber and Newman 1976, Jenkins et al. 1981, Martinoia et al. 1983, Woolhouse 1984) and kinetin-treated leaves (Mittelheuser and Van Stevenink 1971, Venkatarayappa et al. 1984). Overall, it is now an evidence that tissues senescence is a consequence of cumulative membrane deterioration due to increasing level of lipid peroxidation (Dhindsa et al. 1981, Lynch and Thompson 1984) and free radicals (Lynch and Thompson 1984).

We wish to express our gratitude to Prof. G. Dodin, University of Paris VII (Paris) for his many helpful discussions and comments on the mechanistic of free radicals. The authors express their thanks to Dr. P. Rustin for constructive discussions.

\section{References}

Andersson, B., C. Sundby, H.-E. Akerlund and P.-E. Albertsson (1985) Inside-out thylakoid vesicles. An important tool for the characterization of the photosynthetic membrane. Physiol. Plant. 65: 322-330.

Brody, S. S. (1983) Spectral changes in chloroplast during aging. Photochem. Photobiol. 37: 585-586.

Brown, S. B. and P. Jones (1968) Reactions between haemin and hydrogen peroxide. Part 2. Destructive oxidation of haemin. Trans. Faraday Soc. 64: 994-998.

Cohen, B.-S., S. Grossman, A. Pinsky and B. P. Klein (1984) Chlorophyll inhibition of lipoxygenase in growing pea plants. J. Agric. Food Chem. 32: 516-519.

Constantopoulos, G. and C. N. Kenyon (1968) Release of free fatty acids and loss of Hill activity by ageing spinach chloroplasts. Plant Physiol. 43: 531-536.

Dhindsa, R. S., P. Plumb-Dhindsa and T. A. Thorpe (1981) Leaf senescence: Correlated with increased levels of membrane permeability and lipid peroxidation, and decreased levels of superoxide dismutase and catalase. J. Exp. Bot. 32: 93-101.

Douillard, R. (1981) Hypothèses sur la localisation et le role des lipoxygénases des cellules végétales. Physiol. Vég. 19: $533-542$.

Dupont, J. (1983) Contribution à l'étude du vieillissement des mitochondries végétales isolées: Effet de la dégradation des lipides sur les transporteurs d'électrons. Doctoral Thesis, Université Pierre et Marie Curie, Paris, France.

Dupont, J., P. Rustin and C. Lance (1982a) Interaction between mitochondrial cytochromes and linoleic acid hydroperoxide. Possible confusion with lipoxygenase and alternative pathway. Plant Physiol. 69: 1308-1314.

Dupont, J., P. Rustin and C. Lance (1982b) Effect of linoleic acid hydroperoxide on membrane cytochromes of plant mitochondria. Europ. Bioenergetics Conf. Rep. 2: 233-234.

Dupont, J. and P.-A. Siegenthaler (1985) Alterations in the cytochrome composition of spinach thylakoid membranes induced by aging in vitro. J. Plant Physiol. 119: 347-357.

Egmond, M. R., G. A. Veldink, J. F. G. Vliegenthart and J. Boldingh (1973) C-11 H-abstraction from linoleic acid, the rate-limiting step in lipoxygenase catalysis. Biochim. Biophys. Res. Commun. 54: 1178-1184.

Falk, J. E. (1964) Porphyrins and Metalloporphyrins: Their general, physical and coordination chemistry, and laboratory methods. pp. 3-30. Elsevier Publishing Company, Amsterdam.

Fragata, M. (1975) Effects of aging on chlorophyll fluorescence and photosystem II electron transport in isolated chloroplasts. Can. J. Bot. 53: 2842-2845.

Galliard, T. and H. W.-S. Chan (1980) Lipoxygenases. In The Biochemistry of Plants. Edited by P. K. Stumpf. Vol. IV, pp. 133-161. Academic Press, New York.

Grossman, S., B. P. Klein, B. Cohen, D. King and A. Pinsky (1984) Methyl mercuric iodide modification of lipoxygenase-1. Effect on the anaerobic reaction and pigment bleaching. Biochim. Biophys. Acta 793: 455-462.

Heath, R. L. and L. Packer (1968) Photoperoxidation in isolated chloroplasts I. Kinetics and stoichiometry of fatty acid peroxidation. Arch. Biochem. Biophys. 125: 189-198. 
Henry, L. E. A., R. J. Strasser and P.-A. Siegenthaler (1982) Alterations in the acyl lipid composition of thylakoids induced by aging and its effect on thylakoid structure. Plant Physiol. 69: 531-536.

Hoshina, S., T. Kaji and K. Nishida (1975) Photoswelling and light-inactivation of isolated chloroplasts. I. Change in lipid content in light-aged chloroplasts. Plant Cell Physiol. 16: 465-474.

Huber, D. J. and D. W. Newman (1976) Relationships between lipid changes and plastid ultrastructural changes in senescing and regreening soybean cotyledons. J. Exp. Bot. 27: 490-511.

Hynninen, P. H. (1981) Mechanism of the allomerization of chlorophyll: inhibition of the allomerization by carotenoid pigments. Z. Naturforsch. 36B: 1010-1016.

Ikediobi, C. O. and H. E. Snyder (1977) Cooxidation of $\beta$-carotene by an isoenzyme of soybean lipoxygenase. J. Agric. Food Chem. 25: 124-127.

Imamura, M. and S. Shimizu (1974) Metabolism of chlorophyll in higher plants IV. Relationship between fatty acid oxidation and chlorophyll bleaching in plant extracts. Plant Cell Physiol. 15: 187-190.

Ingold, K. U. (1961) Inhibition of the autoxidation of organic substances in the liquid phase. Chem. Rev. 61:563589.

Jenkins, G. I., N. R. Baker and H. W. Woolhouse (1981) Changes in chlorophyll content and organization during senescence of the primary leaves of Phaseoltes vulgaris L. in relation to photosynthetic electron transport. J. Exp. Bot. 32: 1009-1020.

Kato, M. and S. Shimizu (1985) Chlorophyll metabolism in higher plants VI. Involvement of peroxidase in chlorophyll degradation. Plant Cell Physiol. 26: 1291-1301.

Klein, B. P., S. Grossman, D. King, B.-S. Cohen and A. Pinsky (1984) Pigment bleaching, carbonyl production and antioxydant effects during the anaerobic lipoxygenase reaction. Biochim. Biophys. Acta 793: 72-79.

Lynch, D. V. and J. E. Thompson (1984) Lipoxygenase-mediated production of superoxide anion in senescing plant tissues. FEBS Lett. 173: 251-254.

Lüthy, B., E. Martinoia, Ph. Matile and H. Thomas (1984) Thylakoid-associated "chlorophyll oxidase": Distinction from lipoxygenase. Z. Pflanzenphysiol. 113: 423-434.

Mansfield, R. W. and D.S. Bendall (1983) Cytochrome distribution across chloroplast thylakoid membranes controlled proteolysis of inside-out and right-side out vesicles. Biochim. Biophvs. Acta 724: $123-127$.

Martinoia, E., M.J. Dalling and Ph. Matile (1983) Catabolism of chlorophyll: Demonstration of chloroplastlocalized peroxidative and oxidative activities. Z. Pflanzenphysiol. 107: 269-279.

Martinoia, E., U. Heck, M. J. Dalling and Ph. Matile (1983) Changes in chloroplast number and chloroplast constituents in senescing barley leaves. Biochem. Physiol. Pflanzen 178: 147-155.

Merzliak, M. N. and B. A. Koorighnih (1984) Chlorophyll allomerization in the systems containing free radicals, and under illumination of isolated chloroplasts. Studia Biophysica 102: 81-88.

Merzliak, M. N., O. V. Plakunova, S. A. Gotimskij, V. B. Rumyantseva and K. Novak (1984) Lipid peroxidation in and photodamage to a light sensitive chlorescence pea mutant. Physiol. Plant. 62: 329-334.

Mittelheuser, C. J. and R.F. M. Van Stevenink (1971) The ultrastructure of wheat leaves. I. Changes due to natural senescence and the effects of kinetin and ABA on detached leaves incubated in the dark. Protoplasma 73: 239-252.

Okamoto, T. and S. Katoh (1977a) Linoleic acid binding by chloroplasts. Plant Cell Physiol. 18: 539-550.

Okamoto, T., S. Katoh and S. Murakami (1977b) Effect of linoleic acid on spinach chloroplast structure. Plant Cell Physiol. 18: 551-560.

Ortiz, W. and R. Malkin (1985) Topographical studies of the polypeptide subunits of the thylakoid cytochrome $b_{6}-f$ complex. Biochim. Biophys. Acta 808: 164-170.

Peiser, G. D. and S. F. Yang (1977) Chlorophyll destruction by the bisulfite-oxygen system. Plant Physiol. 60: 277281.

Peiser, G. D. and S. F. Yang (1978) Chlorophyll destruction in the presence of bisulfite and linoleic acid hydroperoxide. Phytochemistry 17: 79-84.

Rabek, J. F. and B. Ranby (1978) The role of singlet oxygen in the photooxidation of polymers. Photochem. Photobiol. 28: 557-570.

Russell, G. A. (1959) Fundamental processes of autoxidation. J. Chem. Educ. 36: 111-118.

Rustin, P., J. Dupont and C. Lance (1982) Specific and rapid measurement of lipoxygenase activity in biological materials. Physiol. Vig. 20: 721-727.

Rustin, P., J. Dupont and C. Lance (1984) Involvement of lipid peroxy radicals in the cyanide-resistant electron transport. Physiol. Vég. 22: 643-663. 
Sakaki, T., N. Kondo and K. Sugahara (1983) Breakdown of photosynthetic pigments and lipids in spinach leaves with ozone fumigation: Role of active oxygen. Physiol. Plant. 59: 28-34.

Siegenthaler, P.-A. (1968) Synergic effect of light and aging on the swelling and photochemical activities of isolated chloroplasts. Experientia 24: 1198-1199.

Siegenthaler, P.-A. (1969) Vieillissement de l'appareil photosynthétique. II. Effet synergique de la lumière et du vieillissement sur les activités photochimiques de chloroplastes isolés d'Epinard. Plant Cell Physiol. 10: 811-820.

Siegenthaler, P.-A. (1972) Aging of the photosynthetic apparatus. IV. Similarity between the effects of aging and unsaturated fatty acids on isolated spinach chloroplasts as expressed by volume changes. Biochim. Biophys. Acta 275: 182-191.

Siegenthaler, P.-A. and F. Depéry (1977) Aging of the photosynthetic apparatus. VI. Changes in pH dependence of $\Delta \mathrm{pH}$, thylakoid internal $\mathrm{pH}$ and proton uptake and relationships to electron transport. Plant Cell Physiol. 18: 1047-1055.

Siegenthaler, P.-A. and A. Rawyler (1977) Aging of the photosynthetic apparatus. V. Change in pH dependence of electron transport and relationships to endogenous free fatty acids. Plant Sci. Lett. 9: 265-273.

Takahama, U. (1983) Suppression of lipid photoperoxydation by quercitin and its glycosides in spinach chloroplasts. Photochem. Photobiol. 38: 363-367.

Thomas, H., B. Lüthy and Ph. Matile (1985) Leaf senescence in a non-yellowing mutant of Festuca pratensis Huds. Oxidative chlorophyll bleaching by thylakoid membranes during senescence. Planta 164: 400-405.

Van Rensen, J. J. S. (1975) Lipid peroxidation and chlorophyll destruction caused by diquat during photosynthesis in Scenedesmus. Physiol. Plant. 33: 42-46.

Venkatarayappa, T., R. A. Fletcher and J. E. Thompson (1984) Retardation and reversal of senescence in bean leaves by benzyladenine and decapitation. Plant Cell Physiol. 25: 407-418.

Walling, C. (1963) Chemistry of the organic chemistry. Radiation Res. Supl. 3: 3-16.

Weber, F. and W. Grosch (1976) Co-oxidation of a carotenoid by the enzyme lipoxygenase: Influence on the formation of linoleic acid hydroperoxides. Z. Lebensm. Unters.-Forsch. 161:223-230.

Woolhouse, H. W. (1984) The biochemistry and regulation of senescence in chloroplasts. Can. J. Bot. 62: 29342942.

Yamashita, K., K. Konishi, M. Itoh and K. Shibata (1969) Photobleaching of carotenoids related to the electron transport in chloroplasts. Biochim. Biophys. Acta 172: 511-524.

(Received October 11, 1985; Accepted January 24, 1986) 